\section{Fatores determinantes dos níveis de hemoglobina em crianças aos 12 meses de vida na Zona da Mata Meridional de \\ Pernambuco}

\section{Determinant factors of haemoglobin levels in 12 months old infants in the South of the \\ Zona da Mata of Pernambuco}

Ana Claudia V. M. de S. Lima 1

Pedro Israel Cabral de Lira 2

Sylvia de Azevedo Mello Romani 3

Sophie Helena Eickmann 4

Maria Dilma Piscoya 5

Marília de Carvalho Lima 6

${ }^{1}$ Departamento de Terapia Ocupacional. Universidade Federal de Pernambuco. Rua Prof. Moraes Rego, s. n. Cidade Universitária. Recife, PE, Brasil. CEP: 50.670-901

2,3 Departamento de Nutrição. Universidade Federal de Pernambuco. Recife, PE, Brasil

4-6 Departamento Materno Infantil. Universidade Federal de Pernambuco. Hospital das Clínicas. Recife, PE, Brasil

\begin{abstract}
Objectives: to evaluate factors determining hemoglobin levels in 12 months old infants living in four small towns located in the South of Pernambuco.

Methods: a cross-sectional study conducted in a sub-sample of 245 infants belonging to a cohort of 652 children. Data collection was performed from January to August 1999.

Results: the prevalence of anemia was $73.2 \%$ and mean hemoglobin level $9.8 \mathrm{~g} / \mathrm{dL}(S D=1.6 \mathrm{~g} / \mathrm{dL})$. Variance analysis indicated a significant association between hemoglobin levels and maternal education, absence of a television set at home, birth weight, duration of exclusive breast-feeding, occurrence of diarrhea and nutritional indicators (weight-for-age and length-for-age). Multiple linear regression analysis indicated that socio-economic conditions, birth weight, exclusive breast feeding duration and occurrence of diarrhea had a significant impact on hemoglobin levels.

Conclusions: results confirm that anemia is a severe public health problem, especially during childhood, with multiple factors that contribute to the condition. There is a need to identify the prevailing ones so they can be addressed by programs targeting child health.
\end{abstract}

Key words Anaemia iron-deficiencey, Hemoglobins, Infant, Risk factors

\section{Resumo}

Objetivos: avaliar os fatores determinantes dos níveis de hemoglobina de crianças aos 12 meses de vida, em quatro municípios da Zona da Mata Meridional de Pernambuco.

Métodos: estudo transversal, realizado em uта sub-amostra de 245 lactentes, pertencentes a uma coorte de 652 crianças. A coleta de dados foi realizada no período de janeiro a agosto de 1999.

Resultados: a prevalência de anemia foi de $73,2 \%$, sendo a média de hemoglobina de $9,8 \mathrm{~g} / \mathrm{dL}$ $(D P=1,6 \mathrm{~g} / \mathrm{dL})$. A análise de variância apresentou uma associação estatisticamente significante entre níveis de hemoglobina e escolaridade materna, posse de televisão no domicílio, peso ao nascer, duração do aleitamento materno exclusivo, ocorrência de diarréia e estado nutricional segundo os índices peso/idade e comprimento/idade. A análise de regressão linear múltipla mostrou que as condições socioeconômicas, o peso ao nascer, a duração do aleitamento materno exclusivo e a ocorrência de diarréia tiveram um impacto significante na variação dos níveis de hemoglobina.

Conclusões: os resultados confirmam ser a anemia um relevante problema de saúde pública, especialmente em lactentes, tendo múltiplos fatores que contribuem para o seu surgimento, sendo necessário, portanto, identificar os mais importantes para serem levados em consideração nos programas de saúde da criança.

Palavras-chave Anemia ferropriva, Hemoglobinas, Lactente, Fatores de risco 


\section{Introdução}

A deficiência de ferro, em termos de magnitude, constitui na atualidade o principal problema carencial em escala de saúde pública do mundo.1-3 De acordo com Viteri e Torun, ${ }^{4}$ estima-se que quase dois bilhões de pessoas estejam afetadas pela carência de ferro, em diferentes graus de intensidade, correspondendo a aproximadamente $36 \%$ da população mundial.

A anemia nutricional, especialmente a ferropriva, acomete as populações de quase todos os países do mundo, embora os países mais pobres sejam os mais atingidos, tendo como principais grupos de risco os lactentes, as crianças e adolescentes, as mulheres em idade reprodutiva e indivíduos com dieta insuficiente quantitativa e qualitativamente. 1,2

A anemia por deficiência de ferro em crianças é uma preocupação crescente no Brasil, sendo considerada o principal problema nutricional em menores de cinco anos e que ainda está longe de ser resolvido, 5-8 apesar de todo conhecimento acumulado sobre o assunto e, consequentemente, de serem bastante divulgadas as medidas de intervenção do grupo de consultoria internacional, International Vitamin A Consultative Group (IVACG), sobre anemias (suplementação de ferro, educação alimentar, saneamento básico, assistência à saúde e fortificação de alimentos). 7

Os dados disponíveis demonstram alta prevalência de anemia, variando segundo as diferentes regiões do Brasil, estratos sociais e faixas etárias. 5 Estudos pontuais revelam que a proporção de anêmicos entre as crianças menores de dois anos, situa-se entre $50 \%$ e $83,5 \%$. No entanto, por meio desses estudos, tem-se observado nas últimas décadas um aumento significativo da prevalência e da gravidade da anemia ferropriva nos grupos de risco, em todas as regiões do país, independente de seu nível socioeconômico.9-11

De acordo com Santos, 5 em diferentes regiões e estados do Brasil, no período de 1980 a 1999, foram realizados 73 estudos sobre a distribuição espacial da anemia ferropriva. No entanto, cabe registrar que a maioria destes estudos tem se limitado ao diagnóstico e distribuição espacial dessa carência, com amostras não representativas, viciadas e/ou de tamanho inadequado que se originam de dados de serviços de saúde. Também são escassos os estudos realizados sobre os fatores de risco e, quando eles existem, consideram o problema com relação às variáveis biológicas que estão associadas a sua presença, desconsiderando as variáveis sociais e culturais também definidoras do processo saúde/doença, as quais poderiam proporcionar subsídios para formulação de políticas de saúde e nutrição, visando solucionar o problema e, consequentemente, melhorar a qualidade de vida das crianças. 12,13

Devido a essas questões, este estudo tem como objetivo avaliar os fatores determinantes dos níveis de hemoglobina em lactentes aos 12 meses, residentes em quatro municípios da Zona da Mata Meridional de Pernambuco.

\section{Métodos}

O desenho do estudo foi do tipo transversal, realizado em uma sub-amostra de 245 crianças, pertencentes a uma coorte de 652 crianças do Projeto Saúde e Nutrição Infantil, desenvolvido pelos Departamentos de Nutrição e Materno Infantil da Universidade Federal de Pernambuco. As mesmas foram acompanhadas do nascimento aos 18 meses de vida e residiam nas áreas urbanas de quatro municípios Palmares, Catende, Água Preta e Joaquim Nabuco da Zona da Mata Meridional de Pernambuco, na Região Nordeste do Brasil. Os municípios distam em torno de $120 \mathrm{~km}$ da capital do Estado, Recife, com uma população total de aproximadamente 135.000 habitantes, dos quais, cerca de 20.000 estão na faixa de menores de cinco anos. Desses, $20 \%$ são de crianças com idades inferiores a um ano. 14

A principal atividade econômica da Região é a produção da cana de açúcar. Segundo o último Censo Demográfico, $81 \%$ das famílias recebiam menos de dois salários mínimos (SM) mensais, sendo que $75 \%$ delas recebiam menos de um SM mensal. O analfabetismo, entre os maiores de 15 anos, atingia $26 \%$ das mulheres. 14

O recrutamento das 652 crianças foi realizado no período de setembro de 1997 a agosto de 1998 nas seis maternidades das referidas cidades, as quais cobrem aproximadamente $90 \%$ dos partos. Para atender aos objetivos da pesquisa, a amostra selecionada obedeceu ao seguinte critério de inclusão: intenção de residir na mesma área durante os 18 meses subsequentes ao nascimento e, como critérios de exclusão, a gemelaridade, as anomalias congênitas e os problemas neurológicos ou outras complicações ocorridas na ocasião do parto.

Todas as crianças da coorte nascidas entre janeiro e agosto de $1998(\mathrm{n}=245)$ foram selecionadas para este estudo. O tamanho da amostra foi estimado a partir da identificação das freqüências dos possíveis fatores associados aos níveis de hemoglobina (escolaridade da mãe, bens de consumo, peso ao nascer, aleitamento materno, estado nutricional 
e diarréia) com base em dados da literatura. ${ }^{6,8}$ Considerando-se um erro alfa de $5 \%$, um poder do estudo de $80 \%$, com uma diferença estimada nos níveis de hemoglobina de 0,50 a $1,20 \mathrm{~g} / \mathrm{dL}$ entre os estratos estudados e adotando a fórmula para diferença de médias, 15 calculou-se um número mínimo de 28 crianças para cada estrato.

Através de entrevistas com as mães logo após o parto, utilizando-se questionário padronizado e précodificado, foram obtidas informações sobre as condições socioeconômicas, demográficas, ambientais, maternas e da criança ao nascer.

Os recém-nascidos tiveram as medidas antropométricas (peso e comprimento) e a idade gestacional avaliadas nas primeiras 24 horas de vida, por dois assistentes de pesquisa (enfermeira e nutricionista). Para avaliação da idade gestacional, foi adotado o método de Capurro et al. 16 Para aferição do peso e do comprimento do recém-nascido foram utilizados equipamentos e técnicas padronizadas, obedecendo aos procedimentos estabelecidos pela World Health Organization (WHO). ${ }^{17}$ Foram usadas balanças pesa-bebê (Filizola, modelo digital, 15/2B, São Paulo, Brasil) com precisão de $10 \mathrm{~g}$, e antropômetro de madeira com amplitude de $130 \mathrm{~cm}$ e sub-divisões de $0,1 \mathrm{~cm}$.

O acompanhamento da morbidade e da alimentação das crianças, ao longo dos 12 meses, foi realizado através de visitas domiciliares duas vezes por semana, por uma equipe de 15 entrevistadores. As informações eram prestadas pelas mães e incluíam ocorrência de diarréia, aleitamento materno e introdução de líquidos (água, chás, sucos) e de leite artificial.

Considerou-se aleitamento materno exclusivo quando a criança recebia leite materno diretamente do seio ou extraído, não recebendo qualquer outro alimento sólido ou líquido, incluindo leite não humano. Em relação à diarréia, considerou-se a presença ou não da doença. Duas supervisoras asseguravam diariamente a qualidade das informações que eram analisadas em reuniões semanais com toda a equipe de campo. Quando ocorria a presença das situações mencionadas acima, eram adotados critérios para encaminhamento a assistência médica.

As medidas de peso e comprimento aos 12 meses de vida da criança foram realizadas durante as visitas às residências, por duas antropometristas especialmente treinadas, utilizando equipamentos e técnicas padronizadas, obedecendo aos procedimentos estabelecidos pela WHO. 17 Utilizaram-se balanças de 10 e 25 kg (Modelo MP10 e MP25, CMS Ltda., Londres, Reino Unido), com precisão de $10 \mathrm{~g}$, antropômetro de madeira com amplitude de $130 \mathrm{~cm}$ e sub-divisões de $0,1 \mathrm{~cm}$. O estado nutricional foi avaliado através dos indicadores peso/idade e comprimento/idade utilizando-se o padrão do National Center for Health Statistics (NCHS) como referência, e o ponto de corte de $<-2$ escores $z$ para classificar a desnutrição. ${ }^{17}$

Os níveis de hemoglobina foram avaliados, também aos 12 meses, através de coleta de sangue capilar utilizando-se o Hemocue por um técnico devidamente treinado. As crianças que apresentaram níveis de hemoglobina abaixo de $11 \mathrm{~g} / \mathrm{dL}$ foram consideradas anêmicas 18 e suplementadas com $45 \mathrm{mg}$ de ferro elementar em dose única semanal por seis meses.

As exposições (variáveis independentes) analisadas foram: renda familiar, alfabetização e escolaridade materna, número de pessoas/cômodo, posse de televisão, condições de moradia (tipo de parede e abastecimento de água), idade gestacional, peso ao nascer, sexo, aleitamento materno exclusivo e ocorrência de diarréia do nascimento aos 12 meses, e estado nutricional aos 12 meses. O desfecho analisado (variável dependente) foi o nível de hemoglobina $(\mathrm{g} / \mathrm{dL})$.

Os dados coletados foram codificados e registrados em formulários específicos. Após revisão e codificação, foram os mesmos digitados em dupla entrada, em banco construído com o Programa Epi-info, versão 6,04. As análises estatísticas foram realizadas com o Statistical Package for the Social Sciences, versão 8.0 para Windows.

$\mathrm{O}$ teste $t \mathrm{e}$ a análise de variância (ANOVA) foram os testes estatísticos empregados para comparação das médias na análise bivariada, em virtude da variável dependente ter uma distribuição simétrica.

Previamente à regressão linear múltipla, foi determinado o nível de correlação entre as variáveis independentes através da correlação de Pearson, como forma de excluir variáveis colineares $(r>0,80)$. As variáveis renda per capita e a escolaridade materna foram transformadas em variáveis indicadoras (dum$m y)$, sendo as demais dicotômicas com exceção do peso ao nascer, que foi analisada como variável contínua. Todas as variáveis com $p<0,20$ na análise bivariada foram inicialmente incluídas na análise, com exceção da renda per capita, que pela sua plausibilidade em relação a variável dependente é freqüentemente referida como possível fator determinante dos níveis de hemoglobina.

A estratégia analítica empregada para a análise multivariada foi do tipo hierárquica utilizando-se o método de seqüência "forçada" (enter), segundo os procedimentos sugeridos por Victora et al.,19 e Lima et al. $20 \mathrm{~A}$ abordagem hierárquica consiste na entrada das variáveis explanatórias no modelo em uma or- 
dem previamente estabelecida pelo pesquisador, baseada nas relações lógicas e teóricas entre os fatores determinantes. Para tanto, as variáveis foram agrupadas em três blocos, socioeconômicos/ambientais, da criança ao nascer e durante o primeiro ano de vida, segundo a precedência com que poderiam atuar sobre os níveis de hemoglobina. Do primeiro bloco, das variáveis socioeconômicas/ambientais, fizeram parte, a renda per capita da família, a alfabetização e escolaridade materna, o número de pessoas por cômodo, o bem de consumo televisão, o tipo de construção da residência (parede) e a condição do abastecimento de água. O segundo bloco foi composto pelas variáveis da criança ao nascer, peso, sexo e idade gestacional e o terceiro bloco constou das variáveis da criança durante o primeiro ano de vida, aleitamento materno exclusivo, diarréia e estado nutricional. As variáveis que apresentaram um valor de $\mathrm{p}<0,20$ foram retidas no modelo final da regressão linear.

A pesquisa foi aprovada pelo Comitê de Ética em Pesquisa do Centro de Ciências da Saúde da Universidade Federal de Pernambuco.

\section{Resultados}

Os resultados referem-se as 245 crianças avaliadas quanto ao nível de hemoglobina ( $\mathrm{Hb})$ aos 12 meses de idade, cuja prevalência de anemia foi de 73,5\% com uma média de hemoglobina de 9,8 g/dL [(DP = 1,6 $\mathrm{g} / \mathrm{dL}$ ). Entre essas, $28,2 \%$ tinham valores inferiores a

Tabela 1

Características socioeconômicas e ambientais das famílias e médias de hemoglobina de crianças aos 12 meses de idade residentes na Zona da Mata Meridional de Pernambuco, 1999.

\begin{tabular}{|c|c|c|c|c|c|c|}
\hline \multirow{2}{*}{ Variáveis } & \multicolumn{2}{|c|}{ Total } & \multicolumn{2}{|c|}{ Hemoglobina } & \multirow{2}{*}{ IC95\% } & \multirow{2}{*}{$\begin{array}{c}\text { Análise } \\
\text { estatística* }\end{array}$} \\
\hline & $N=245$ & $\%$ & Média & DP & & \\
\hline \multicolumn{7}{|l|}{ Socioeconômicas } \\
\hline \multicolumn{7}{|c|}{ Renda familiar per capita } \\
\hline$\leq 0,25$ (SM) & 64 & 26,0 & 9,62 & 1,69 & $9,20-10,05$ & \\
\hline $0,25-0,50$ & 78 & 32,0 & 9,71 & 1,64 & $9,35-10,09$ & $F=0,66$ \\
\hline$>0,50(\mathrm{SM})$ & 103 & 42,0 & 9,90 & 1,56 & $9,60-10,21$ & $p=0,51$ \\
\hline \multicolumn{7}{|l|}{ Alfabetização da mãe } \\
\hline Analfabeta & 47 & 19,0 & 9,47 & 1,79 & $8,95-10,06$ & $t=1,91$ \\
\hline Alfabetizada & 198 & 81,0 & 9,84 & 1,57 & $9,62-10,06$ & $p=0,17$ \\
\hline \multicolumn{7}{|l|}{ Escolaridade materna } \\
\hline$\leq 4$ anos & 89 & 36,0 & 9,46 & 1,78 & $9,08-9,83$ & $F=3,93$ \\
\hline $5-8$ anos & 98 & 40,0 & 9,78 & 1,48 & $9,48-10,08$ & $p=0,02$ \\
\hline$\geq 9$ anos & 58 & 24,0 & 10,22 & 1,50 & $9,82-10,61$ & \\
\hline \multicolumn{7}{|l|}{ Número de pessoas/cômodo } \\
\hline$\leq 03$ & 162 & 66,0 & 9,88 & 1,65 & $9,62-10,14$ & $t=2,25$ \\
\hline$>3$ & 83 & 34,0 & 9,55 & 1,54 & $9,21-9,89$ & $p=0,13$ \\
\hline \multicolumn{7}{|l|}{ Televisão } \\
\hline Não & 25 & 10,0 & 8,96 & 1,58 & $8,31-9,61$ & $t=7,08$ \\
\hline Sim & 220 & 90,0 & 9,86 & 1,60 & $9,65-10,07$ & $p=0,008$ \\
\hline \multicolumn{7}{|l|}{ Ambientais } \\
\hline \multicolumn{7}{|l|}{ Parede } \\
\hline Alvenaria & 207 & 84,5 & 9,85 & 1,51 & $9,64-10,06$ & $t=3,55$ \\
\hline Taipa/tábua/papelão & 38 & 15,5 & 9,31 & 2,09 & $8,63-10,07$ & $p=0,06$ \\
\hline \multicolumn{7}{|l|}{ Abastecimento de água } \\
\hline Canalização interna & 216 & 88,0 & 9,82 & 1,57 & $9,61-10,03$ & $t=1,80$ \\
\hline Sem canalização & 29 & 12,0 & 9,39 & 1,91 & $8,66-10,12$ & $p=0,18$ \\
\hline
\end{tabular}

* $F$, ANOVA; $t$, teste " $t$ " de Student 
9,0 g/dL, estando incluídos nesse grupo 4,9\% com níveis de hemoglobina inferiores a 7,0 g/dL.

As características socioeconômicas e ambientais da população estudada são apresentadas na Tabela 1 . Em geral as famílias eram pobres, com cerca de $2 / 3$ percebendo uma renda familiar per capita inferior a de 0,5 salário mínimo/mês, equivalente a US\$50 (R\$75,00); 36\% das mães apresentavam com de escolaridade $\leq 4$ anos e em $34 \%$ das residências moravam mais de três pessoas/cômodo. Apenas 10\% das famílias não possuíam televisão, em $15,5 \%$ das casas, as paredes eram de taipa, tábua ou papelão e em $12 \%$ não havia canalização interna de abastecimento d'água.

A Tabela 2 apresenta as variáveis da criança ao nascer e do nascimento aos 12 meses de vida. Observa-se que $9,4 \%$ nasceram com baixo peso e $9,8 \%$ apresentaram idade gestacional $<37$ semanas. Durante o primeiro ano de vida, $72,7 \%$ das crianças receberam leite materno exclusivo por menos de 20

Tabela 2

Características ao nascimento e do nascimento aos 12 meses de vida e médias de hemoglobina de crianças residentes na Zona da Mata Meridional de Pernambuco, 1999.

\begin{tabular}{|c|c|c|c|c|c|c|}
\hline \multirow{2}{*}{ Variáveis } & \multicolumn{2}{|c|}{ Total } & \multicolumn{2}{|c|}{ Hemoglobina } & \multirow{2}{*}{ IC95\% } & \multirow{2}{*}{$\begin{array}{c}\text { Análise } \\
\text { estatística* }\end{array}$} \\
\hline & $N=245$ & $\%$ & Média & DP & & \\
\hline \multicolumn{7}{|l|}{ Ao nascer } \\
\hline \multicolumn{7}{|l|}{ Sexo } \\
\hline Masculino & 121 & 49,0 & 9,62 & 1,61 & $9,33-9,91$ & $t=2,02$ \\
\hline Feminino & 124 & 51,0 & 9,91 & 1,61 & $9,63-10,20$ & $p=0,15$ \\
\hline \multicolumn{7}{|l|}{ Peso $(g)$} \\
\hline$\leq 2499$ & 23 & 9,4 & 8,51 & 2,10 & $7,60-9,42$ & \\
\hline 2500-2999 & 79 & 32,2 & 9,70 & 1,56 & $9,35-10,05$ & $F=9,05$ \\
\hline$\geq 3.000$ & 143 & 58,4 & 10,01 & 1,47 & $9,76-10,25$ & $p<0,001$ \\
\hline \multicolumn{7}{|c|}{ Idade gestacional (semanas) } \\
\hline$<37$ & 24 & 9,8 & 8,74 & 1,86 & $7,95-9,52$ & $t=11,18$ \\
\hline$\geq 37$ & 221 & 90,2 & 9,88 & 1,55 & $9,67-10,09$ & $p=0,01$ \\
\hline \multicolumn{7}{|c|}{ Do nascimento aos 12 meses } \\
\hline \multicolumn{7}{|c|}{ Aleitamento materno exclusivo (dias) } \\
\hline$<20$ & 178 & 72,7 & 9,59 & 1,67 & $9,34-9,83$ & $t=8,47$ \\
\hline$\geq 20$ & 67 & 27,3 & 10,25 & 1,37 & $9,92-10,59$ & $p=0,04$ \\
\hline \multicolumn{7}{|l|}{ Diarréia (dias) } \\
\hline Não & 85 & 34,7 & 10,13 & 1,60 & $9,79-10,48$ & $t=6,80$ \\
\hline Sim & 160 & 65,3 & 9,57 & 1,60 & $9,32-9,82$ & $p=0,01$ \\
\hline \multicolumn{7}{|l|}{ Aos 12 meses } \\
\hline \multicolumn{7}{|c|}{ Estado nutricional (escore $z$ ) } \\
\hline \multicolumn{7}{|l|}{ Peso/idade } \\
\hline$<-2$ & 9 & 3,7 & 9,31 & 1,65 & $8,04-10,59$ & \\
\hline$-2 a<-1$ & 66 & 27,0 & 9,26 & 1,76 & $8,84-9,70$ & $F=5,29$ \\
\hline$\geq-1$ & 170 & 69,3 & 9,99 & 1,52 & $9,76-10,22$ & $p=0,005$ \\
\hline \multicolumn{7}{|c|}{ Comprimento/idade } \\
\hline$<-2$ & 21 & 8,6 & 9,03 & 2,04 & $8,10-10,96$ & \\
\hline$-2 a<-1$ & 56 & 22,8 & 9,34 & 1,61 & $8,91-9,78$ & $F=6,10$ \\
\hline$\geq-1$ & 168 & 68,6 & 10,0 & 1,51 & $9,78-10,24$ & $p=0,002$ \\
\hline
\end{tabular}

* F, ANOVA; $t$, teste " $t$ " de Student 
Modelo de regressão linear hierarquizado de grupos de fatores associados à concentração de hemoglobina de crianças aos 12 meses de vida. Zona da Mata Meridional de Pernambuco, 1999.

\begin{tabular}{|c|c|c|c|c|}
\hline \multirow{3}{*}{ Variáveis } & \multicolumn{3}{|c|}{ Modelos } & \multirow{3}{*}{$\mathbf{R}^{2}$} \\
\hline & 1 & 2 & 3 & \\
\hline & $\beta$ & $\beta$ & $\beta$ & \\
\hline \multicolumn{5}{|l|}{ Socioeconômicas/ambientais } \\
\hline Escolaridade (anos) & & & & 3,1 \\
\hline$\leq 4$ & $-0,66^{*}$ & $-0,69 * *$ & $-0,42$ & \\
\hline $5-8$ & $-0,46 f$ & $0,52 *$ & $-0,31$ & \\
\hline Televisão (não) & $-0,79 *$ & $-0,72 *$ & $-0,73^{*}$ & 2,1 \\
\hline \multicolumn{5}{|l|}{ Da criança (ao nascer) } \\
\hline Peso (kilos) & & $0,68 * * *$ & $0,60 * *$ & 3,3 \\
\hline Sexo (masculino) & & $-0,40 *$ & $-0,36 f$ & 1,4 \\
\hline \multicolumn{5}{|l|}{ Assistência à criança } \\
\hline Aleitamento materno exclusivo ( $<20$ dias) & & & $-0,47 *$ & 1,4 \\
\hline \multirow[t]{2}{*}{ Diarréia (Sim) } & & & $-0,41 *$ & 1,4 \\
\hline & & & & 12,7 \\
\hline
\end{tabular}

$\beta=$ Coeficiente de regressão; $R^{2}=$ Coeficiente de determinação; Níveis de significância: $f P \leq 0,10 ; * P \leq 0,05 ; * * P \leq 0,01 ; * * * P \leq 0,001$

dias e a diarréia ocorreu em $65,3 \%$ dos casos. O estado nutricional avaliado aos 12 meses revelou que $3,7 \%$ e $8,6 \%$ apresentaram déficit ( $<-2$ escores $z$ ) de peso e de comprimento para a idade, respectivamente.

As referidas tabelas ainda apresentam os resultados das associações entre as variáveis independentes e os níveis de hemoglobina. Entre as variáveis socioeconômicas e ambientais apenas a escolaridade materna e o bem de consumo televisão estiveram associadas significativamente $(\mathrm{p}<0,05)$. As variáveis da criança como o peso ao nascer, idade gestacional, aleitamento materno exclusivo, ocorrência de diarréia e estado nutricional, apresentaram associação significante com o desfecho.

Os resultados da regressão linear multivariada com os níveis de hemoglobina como variável dependente, constam da Tabela 3. Entre as variáveis socioeconômicas componentes do Modelo 1, a escolaridade materna ( $\leq 4$ anos de estudo) e a não disponibilidade de televisão se mostraram significantes com a concentração de hemoglobina, contribuindo com 5,2\%. O Modelo 2 (das variáveis da criança ao nascimento), revelou uma contribuição de $4,7 \%$, tendo o peso contribuído com 3,2\%. No Modelo 3, as variáveis da criança durante o primeiro ano de vida, aleitamento materno exclusivo por menos de 20 dias e ocorrência de diarréia, se mostraram significativas, contribuindo esse bloco com 2,8\%. Consideradas no seu conjunto, as variáveis explicaram $12,7 \%$ da variação do nível de hemoglobina, permanecendo como significantes no modelo final as variáveis socioeconômicas/ambientais (ausência de televisão), o peso ao nascer, o aleitamento materno exclusivo e a ocorrência de diarréia.

\section{Discussão}

A anemia ferropriva tem sido considerada um relevante problema de saúde pública, especialmente em países em desenvolvimento, onde as crianças menores de cinco anos são mais susceptíveis a fatores de risco biológicos, socioeconômicos, demográficos e ambientais.

O propósito deste estudo foi examinar os possíveis fatores determinantes dos níveis de hemoglobina em crianças aos doze meses de vida na Zona da Mata Meridional de Pernambuco, cuja avaliação identificou uma elevada prevalência de anemia $(73,5 \%)$.

Vários autores têm destacado que a alta 
prevalência de anemia até os dois anos de idade deve-se a uma maior velocidade do crescimento, ao desmame precoce, à falta de uma dieta balanceada com alimentos ricos em ferro e a uma maior prevalência de doenças como diarréias e infecções respiratórias nos primeiros anos de vida. ${ }^{21-23}$

Neste estudo, os determinantes distais foram representados pelas variáveis socioeconômicas/ambientais que se sobrepunham aos determinantes intermediários formados pelas variáveis da criança ao nascer e os proximais aquelas avaliadas durante o primeiro ano de vida (alimentação, morbidade e estado nutricional. As variáveis estudadas através da análise de regressão linear múltipla explicaram $12,7 \%$ da variação dos níveis de hemoglobina aos 12 meses de vida.

A renda per capita não se constitui um fator associado aos níveis de hemoglobina. Esse resultado pode refletir uma homogeneidade da amostra com relação às precárias condições de vida ou o fato da renda não se constituir dentre as variáveis socioeconômicas o único fator a ser considerado, devendose observar outros como educação, saneamento e acesso a serviços de saúde 6 ou a posse de bens de consumo, como no caso do presente estudo, onde a não disponibilidade de televisão no domicílio esteve associada significativamente com uma menor média de hemoglobina.

Estudos anteriores têm referido que a renda per capita é um importante fator determinante da anemia.6,13,24,25 No estudo de Sigulem et al.,26 verificou-se que a proporção de crianças anêmicas é significativamente maior entre aquelas pertencentes a famílias com renda per capita inferior a 1,0 salário mínimo, no entanto, essa associação só foi observada em crianças maiores de 24 meses de idade. Silva et al. 27 constataram que as crianças de famílias com renda per capita de até um salário mínimo tiveram um risco adicional de $57 \%$ de apresentar anemia.

Outros autores relatam que mesmo com o aumento da renda não se garante o aumento dos níveis de hemoglobina, uma vez que esta carência também é freqüente e apresenta-se com risco elevado em populações de melhores níveis socioeconômicos. 28 Portanto, a característica peculiar da anemia com sua disseminação por todos os estratos socioeconômicos da população, reforça a importância dessa deficiência em nosso meio. 26,29

Monteiro et al. ${ }^{6}$ vêm realizando, desde 1984, estudos populacionais com crianças menores de cinco anos na cidade de São Paulo, os quais enfatizam que a prevalência da anemia tende a diminuir com a melhoria do nível socioeconômico, apontando assim, para a necessidade de estudos mais refinados sobre a exata determinação que a condição socioeconômica exerce sobre os níveis de hemoglobina.

A escolaridade materna, que esteve associada significativamente com os níveis de hemoglobina neste estudo, confirma o achado de Osório ${ }^{13} \mathrm{em}$ crianças entre 6-59 meses de vida no estado de Pernambuco. Um recente estudo, realizado em São Paulo por Monteiro et al., 6 com crianças menores de cinco anos também revelou que mesmo com o aumento da escolaridade do chefe da família, ainda é muito alto o risco de anemia. Por outro lado, há trabalhos na literatura que não constataram tal associação. 26,29-31

Após a inclusão das variáveis ambientais ajustadas pela escolaridade materna e disponibilidade de televisão, as mesmas não se mostraram significantes, tendo sido excluídas, confirmando os achados de Monteiro et al., 6 para a cidade de São Paulo, quando se analisou a rede de abastecimento de água e de esgoto. No entanto, outros pesquisadores encontraram associação entre variáveis ambientais, como saneamento, abastecimento de água e coleta de lixo com os níveis de hemoglobina. 13,22,29

Entre as variáveis da criança, o peso ao nascer foi um fator determinante dos níveis de hemoglobina. $\mathrm{Na}$ literatura, o baixo peso ao nascer é um dos fatores predisponentes mais importantes para os baixos níveis, sendo mais acentuado no primeiro ano de vida, por terem as crianças logo após o nascimento, baixas reservas de ferro e por serem desmamadas precocemente, acentuando sua depleção.13,30,32-34 Por outro lado, Silva et al.,27 não observaram associação significativa entre o baixo peso ao nascer e a anemia das crianças de 12 a 18 meses de idade, tendo os mesmos atribuídos a ausência de associação ao pequeno tamanho da amostra.

Em relação ao sexo, o mesmo não se constitui um fator determinante da anemia. Esse achado corrobora os estudos de Torres et al.,22 e Hassan et al. 35

Compondo o terceiro modelo, o aleitamento materno exclusivo demonstrou esta associação ( $p$ $<0,05)$ para as crianças que amamentaram exclusivamente por $<20$ dias. O mesmo foi observado por Torres et al.,22 estudando crianças menores de um ano em São Paulo, que apresentavam menor prevalência de anemia quando amamentadas exclusivamente por um período igual ou superior a seis meses. Outros estudos têm demonstrado resultados semelhantes como os de Saarinem e Siimes, ${ }^{36}$ Garry et al., 37 e Souza. ${ }^{38}$ Por outro lado, em alguns estudos ressalta-se que não foram encontradas associações entre o tempo de amamentação e os níveis de hemoglobina. $21,27,32,39,40$

Monteiro et al., 6 enfatizaram que diferentemente do leite de vaca não modificado ou das fórmulas infantis, o leite materno propicia às crianças ferro de 
alta biodisponibilidade e proteção contra infecções, condições essas que as protegem da anemia. A diarréia esteve associada com os níveis de hemoglobina após ser ajustada por todas as outras variáveis anteriores. Segundo Jansson et al., ${ }^{41}$ é grande a predisposição do surgimento da anemia após um episódio de infecção aguda, variando de acordo com a duração e severidade da doença. Outros estudos mostram que infecções leves ocorrem em cerca de $60 \%$ das crianças entre os 9-12 meses de vida e que essas estão claramente associadas com os baixos níveis de hemoglobina. 42

A anemia ferropriva tem múltiplos fatores que contribuem para o seu surgimento, não estando sua origem apenas atrelada aos fatores biológicos e sim, dentro de um contexto mais amplo, onde se deve evidenciar fatores socioeconômicos, culturais, de morbidade, presentes na população infantil.

Um dos fatores importantes é o consumo alimen- tar, que não foi possível ser contemplado neste trabalho. No estudo de Osório, 13 realizado no Estado de Pernambuco com crianças na faixa etária de 6-59 meses de idade verificou-se que o consumo de ferro não atingiu as recomendações diárias de $10 \mathrm{mg}$ na maioria dos grupos etários em todas as áreas geográficas, sendo o consumo mais baixo em idades menores e no interior rural, atingindo em média, praticamente a metade do que seria recomendado $(0,49 \mathrm{mg})$.

Face ao exposto, através da determinação dos principais fatores associados à anemia ferropriva na faixa etária de menores de um ano, faz-se necessário o estabelecimento de programas de saúde, priorizando uma intervenção efetiva em grupos de risco, através da utilização de medidas básicas como educação da população, educação nutricional, suplementação medicamentosa e fortificação de alimentos com ferro.

\section{Agradecimentos}

Às famílias participantes, aos 22 trabalhadores de campo, à equipe de processadores e digitadores dos dados, ao staff das seis maternidades da área, ao Hospital Regional dos Palmares da Secretaria de Saúde do Estado de Pernambuco, a Coordenação de Aperfeiçoamento de Pessoal de Nível Superior (CAPES) e ao Conselho Britânico. Este projeto foi financiado pelo Instituto Nacional de Alimentação e Nutrição (INAN) do Ministério da Saúde em Processo $\mathrm{n}^{\circ} 25350.001472 / 96$.

\section{Referências}

1. MacPhail P, Bothwell TH. The prevalence and causes of nutritional iron deficiency anemia. In: Fosson SJ, Zlotkins S. Nutritional anemias. New York: Nestlé; 1992. p. 1-12.

2. Batista Filho M, Ferreira LOC. Prevenção e tratamento da anemia nutricional ferropriva: novos enfoques e perspectivas. Cad Saúde Pública 1996; 12: 37-45.

3. UNICEF (Fundo das Nações Unidas para a Infância). Situação mundial da infância. Brasília (DF): O Fundo; 1998.

4. Viteri FE, Torun B. Anaemia and physical work capacity. Clin Haematol 1993; 3: 82-96.

5. Santos LMP. Bibliografia sobre deficiência de micronutrientes no Brasil, 1999-2000: anemia. Brasília (DF): OPAS, OMS; 2002. v. 2

6. Monteiro CA, Szarfarc SC, Mondini L. Tendência secular da anemia na infância de São Paulo (1984-1996). Rev Saúde Pública 2000; 34: 62-72.

7. Queiroz SS, Torres MAA. Anemia ferropriva na infância. J Pediatr [Rio de Janeiro] 2000; 73: 298-304.

8. Osório MM, Lira PIC, Batista-Filho M. Prevalence of anaemia in children 6-59 months old in the state of Pernambuco, Brazil. Rev Panam Salud Publica 2001; 10: 
101-7.

9. Batista Filho M, Rissin A. Deficiências nutricionais: ações específicas do setor saúde para o seu controle. Cad Saúde Pública 1993; 9: 130-5.

10. Torres MAA, Sato K, Juliano Y, Queiroz SS. Terapêutica em doses profiláticas de sulfato ferroso como medida de intervenção no combate à carência de ferro em crianças atendidas em unidades básicas de saúde. Rev Saúde Pública 1994; 28: 410-5.

11. Szarfarc SC, Stefani MLR, Lerner BR. Anemia nutricional no Brasil. Cad Nutrição 1995; 9: 5-24.

12. Martins IS, Alvarenga AT, Siqueira AAF, Szarfarc SC, Lima F. As determinações biológicas e sociais da doença: um estudo de anemia ferropriva. Rev Saúde Pública 1987; 21: 73-89.

13. Osório MM. Fatores determinantes da anemia ferropriva na infância. J Pediatr [Rio de Janeiro] 2002; 78: 269-78.

14. FIBGE (Fundação Instituto Brasileiro de Geografia e Estatística). Censo demográfico: 2001. Rio de Janeiro: A Fundação; 2001.

15. Kirkwood BR. Essentials of medical statistics. London: Blackwell Science; 1988.

16. Capurro H, Konichezky S, Fonseca D, Caldeyro-Barcia R A simplified method for diagnosis of gestational age in the newborn infant. J Pediatr 1978; 93: 120-2.

17. WHO (World Health Organization).Physical status: the use and interpretation of anthropometry. Geneva: The Organization; 1995. (Techinical Report Series, 854).

18. DeMaeyer E. Preventing and controlling iron deficiency anaemia through primary health care. A guide for health administrations and programme managers. Geneva: WHO; 1989.

19. Victora CG, Huttly SRH, Fuchs SC, Olinto MTA. The role of conceptual frameworks in epidemiological analysis: a hierarchical approach. Int J Epidemiol 1997; 26: 224-7.

20. Lima M, Ismail S, Ashworth A, Morris SS. Influence of heavy agricultural work during pregnancy on birthweight in Northeast Brazil. Int J Epidemiol 1999; 28: 469-74.

21. Monteiro CA, Szarfarc SC. Estudo das condições de saúde de crianças do município de São Paulo. Rev Saúde Pública $1987 ; 21: 255-60$

22. Torres MAA, Sato K, Queiroz SS. Anemia em crianças menores de dois anos atendidas nas unidades básicas de saúde no Estado de São Paulo, Brasil. Rev Saúde Pública 1994; 28: 290-4.

23. Schmitz BAS, Picanço MR, Aquino KKNC, Bastos J, Giorgini E, Cardoso R, Braga JAP, Fisberg M. Prevalência de desnutrição e anemia em pré-escolares de Brasília (Brasil). Pediatr Mod 1998; 34: 155-64.

24. Romani SAM, Lira PIC, Batista Filho M, Sequeira LAS, Freitas CLC. Anemia em pré-escolares: diagnóstico, tratamento e avaliação. Arch Latinoam Nutr 1991; 41 $159-67$.

25. Sargent JD, Stukel TA, Dalton MA, Freeman JL, Brown MJ. Iron deficiency in Massachutts communities: socioeconomic and demographic risk factors among children. Am J Public Health 1996; 86: 544-50.
26. Sigulem DM, Tudisco ES, Goldenberg P, Athaide MM, Vaisman E. Anemia ferropriva em crianças do município de São Paulo. Rev Saúde Pública 1978; 12: 168-78.

27. Silva LSM, Giugliani ER, Aerts DRGC. Prevalência e determinantes de anemia em crianças de Porto Alegre, RS, Brasil. Rev Saúde Pública 2001; 35: 66-73.

28. Politt E. Poverty and child development: relevance of research in developing countries to the United States. Child Dev 1994; 65: 283-95.

29. Neumann NA, Tanaka OY, Szarfac SC, Guimarães PRV, Victora CG. Prevalência e fatores de risco para anemia no Sul do Brasil. Rev Saúde Pública 2000; 34: 56-63.

30. Emond AM, Hawkins N, Pennocck C, Golding J. Haemoglobin and ferritin concentrations in infants at 8 months of age. Arch Dis Child 1996; 74: 36-9.

31. Assis AMO, Santos LMP, Martins MC, Araújo MPN, Amorim DQ, Morris SS, Barreto ML. Distribuição da anemia em pré-escolares do semi-árido da Bahia. Cad Saúde Pública 1997; 13: 237-43.

32. Lehmann F, Gray-Donald K, Mongeon M, Tommaso SD Iron deficiency anemia in 1-year-old children of disavantaged families in Montreal. Can Med Assoc 1992; 146 : 1571-7.

33. Cohen JH, Haas JD. The comparison of mixed distribution analysis with a three-criteria model as a method for estimating the prevalence of iron deficiency anaemia in Costa Rica in children aged 12-23 months. Int Epidemiol Assoc 1999; 28: 82-9.

34. Torres MAA, Sato K, Queiroz SS. Efeito do uso do leite fortificado com ferro e vitamina $\mathrm{C}$, sobre os níveis de hemoglobina e condição nutricional de crianças menores de 2 anos. Rev Saúde Pública 1995; 29: 301-7.

35. Hassan K, Sullivan, KM, Yip, R, Woodruff, BA. Factors associated with anemia in refugee children. J Nutr 1997; 127: 2194-8.

36. Saarinem UM, Siimes MA. Iron absorption from breast milk, cow's milk and iron-supplemented formula: an opportunistic use of changes in total body iron determined by hemoglobin, ferritin and body weight in 132 infants. Pediatr Rev 1979; 13: 143-9.

37. Garry PJ, Owen GM, Hooper EM, Gilbert BA. Iron absorption from human milk and formula with and without iron supplementation. Pediatr Rev 1981; 15: 822-9.

38. Souza SB. Anemia e alimentação no primeiro ano de vida. Rev Saúde Pública 1994; 14: 17-20.

39. Souza SB, Szarfac SC, Souza JMP. Anemia no primeiro ano de vida em relação ao aleitamento materno. Rev Saúde Pública 1997; 31: 15-20.

40. Karr M, Alperstein G, Causer J, Mira M, Lammi A, Fett MJ. Iron status and anaemia in preschool children in Sydney. Aust N Z J Public Health 1996; 20: 618-22.

41. Jansson LT, Kling S, Dalmann PR. Anemia in children with acute infections seen in a primary care pediatrics outpatient clinic. Pediatr Infect Dis 1986; 4: 424-7.

42. Reeves JD, Yip R, Kiley VA, Dalmann PR. Iron deficiency in infants: the influency of mild antecedent infection. J Pediatr 1984; 105: 874-9.
Recebido em 4 de agosto de 2003

Versão final reapresentada em 20 de novembro de 2003

Aprovado em 5 de janeiro de 2004 